\title{
Flu Vaccine Coupled to Vitamin D Decrease the Frequency of Lower Respiratory Tract Infections (Randomized Controlled Trial)
}

\author{
C. Tayeh, P. Noun, A. Farah, Y. Khalife, G. Abi Fares, \\ M. Amm, H. Feghali, G. Nicolas, M. C. Fadous Khalife* \\ Holy Spirit University of Kaslik, University Hospital (UH)-Notre Dame Des Secours, Byblos, Lebanon \\ Email: *mclaude454@hotmail.com
}

How to cite this paper: Tayeh, C., Noun, P., Farah, A., Khalife, Y., Abi Fares, G., Amm, M., Feghali, H., Nicolas, G. and Fadous Khalife, M.C. (2017) Flu Vaccine Coupled to Vitamin D Decrease the Frequency of Lower Respiratory Tract Infections (Randomized Controlled Trial). Open Journal of Pediatrics, 7, 353-358.

https://doi.org/10.4236/ojped.2017.74040

Received: November 8, 2017

Accepted: December 25, 2017

Published: December 28, 2017

Copyright (@) 2017 by authors and Scientific Research Publishing Inc. This work is licensed under the Creative Commons Attribution International License (CC BY 4.0).

http://creativecommons.org/licenses/by/4.0/

\begin{abstract}
Introduction: Influenza disease is one of the major health concerns in pediatrics. One of the most recently suggested effects of vitamin D is preventing infectious diseases. The aim of our study was to compare flu vaccine effect to flu vaccine associated to vitamin $\mathrm{D}(100,000$ UI per OS) in reducing respiratory tract infections in children. Patients and Methods: 347 healthy children aged 2 to 5 years randomly selected during fall 2012 and the following season fall 2013 were included; upper and lower respiratory tract infections following vaccination were noted. Our results were analyzed using SPSS program (Chi-square and T-test). Results: Out of a total of 347 children included, 150 received flu vaccine and 197 received flu vaccine plus vitamin D. 88 children from both groups manifested upper respiratory tract infections and 64 had lower respiratory tract infections. The statistical analysis revealed no significant difference between the two groups concerning the upper respiratory tract infection $(p=0.207)$ and a significant difference between groups for the lower respiratory tract infection $(\mathrm{p}=0.001)$. Conclusion: Coupling vitamin $\mathrm{D}$ to flu vaccine provides no protection against upper respiratory infections but provides an effective strategy to decrease the rate of lower respiratory infections in children $2-5$ years of age.
\end{abstract}

\section{Keywords}

Influenza, Flu Vaccine, Vitamin D, Upper and Lower Respiratory Tract

\section{Introduction}

Influenza has long been a major health problem in the general and especially pediatric population that over-goes important seasonal fluctuations. Cannel and $\mathrm{Al}$ 
[1] suggested the hypothesis that seasonal variations of serum vitamin D concentrations can affect the epidemic incidence of the flu.

On one side, vitamin $\mathrm{D}$ can increase the innate immune response and protect against infections of the respiratory tract, but few clinical studies have evaluated the relation between vitamin $\mathrm{D}$ and the seasonal flu. A clinical study has shown a decrease in the seasonal influenza infections in Japanese children receiving 1200 UI of vitamin D3 per day [2]. We have therefore decided to couple a loading dose of vitamin D (100,000 UI per OS) to the flu vaccine, and to compare the results to a group that was administered the vaccine only. We had to observe the effect of both on the incidence of the seasonal flu and upper respiratory tract infections as well as secondary lower respiratory tract infections, over 2 winter seasons from September 2012 till the end of January 2014. The 347 previously healthy children, aged 2 to 5 , were randomly selected out of 3 different pediatric doctors' offices across Lebanon.

\section{Tools and Methods}

We have carried on a multi-centered, prospective study over the course of 2 winter seasons (years 2012-2013 \& 2013-2014) with 347 children of both sexes. It took place in 3 private clinics of 3 pediatricians, taking into consideration variations in climate, humidity, altitude, way of living, eating habits \& more factors that could have a relation with vitamin $\mathrm{D}$ levels. The exclusion criteria were age (under 2 years or over 5 years), chronic intake of vitamin D, history of recent vitamin D supplementation during the last 3 months, history of allergy to the immunization, recent viral, bacterial or parasitic infections, children with chronic respiratory diseases (asthma, cystic fibrosis, etc.), congenital diseases, chronic cardiac, renal, digestive, vascular, endocrine diseases, and finally patients who received recent immunosuppressive or chemotherapeutic agents during the previous year.

Patients who met the inclusion criteria were divided into 2 groups: group A received only the influenza vaccine, group $B$ received both a single dose of Vitamin D 100,000 IU orally coupled with the administration of the Influenza vaccine. Parents were informed of the study goals, possible side effects and follow up. An oral approval was documented before initiating the study. Belonging to a group or the other was performed according to a coin toss. The choice of the dose of 100,000 UI was made according to the European recommendations. Patients were followed up for 3 months only since the vaccine effect is the highest in this period. The age range of 2 to 5 years was chosen because it reflects the period of admission into collectivities, of adenoids hypertrophy and its function as a bacterial/viral reservoir. This role starts to decrease beyond 5 year of age. The exclusion of the younger than 2 years was justified by their daily supplementation.

The vaccine was administered by the intramuscular way. Patients did not suffer from any side effect of the vaccine or vitamin $\mathrm{D}$ during the course of the study. Parents were instructed clearly to inform the physician during the study period for any symptoms including but not limited to rhinorrhea, headache, fever, cough, 
decrease in appetite, nausea, vomiting, and diarrhea as well as various infections divided into two categories: upper respiratory tract infections (otitis, sinusitis, tonsillitis, ethmoiditis, etc.) and lower respiratory tract infections (laryngitis, bronchitis, pneumonia). No child was given more than one dose of vitamin D during the study.

Statistical analysis of the results was executed using the SPSS (version 16.00) program (SPSS Inc., Chicago, IL); the board containing continuous and discrete variables. The discrete variables were analyzed using the Chi square method, the continuous variables using the " $r$ " test. Probability values of $p \leq 0.05$ were considered significant.

\section{Results}

The number of patients included in this study was 417 , male gender was slightly more predominant $(52.2 \%)$. The average age was 3.82 years with a standard deviation of 1.12. The average weight on presentation of the patients was $17.83 \mathrm{~kg}$ with a standard deviation of 5.16.

Group A included 150 children (43.2\%) and group B included 197 children (56.8\%). No statistical significance was noted when comparing the average age, gender distribution and average weight (Table 1).

Patients who had upper respiratory tract infections had similar distribution of gender and average age however had a lower average weight of $17 \mathrm{~kg}(\mathrm{p}=0.018)$ when compared to patients who did not have any upper respiratory tract infection $(18 \mathrm{~kg})$.

Patients who suffered from lower respiratory tract infections were also analyzed in terms of age, weight and gender and no statistically significant difference was noted $(\mathrm{p}=0.184, \mathrm{p}=0.108, \mathrm{p}=0.509$ respectively).

However, significant results were shown on lower respiratory tract infections between the children who received the vaccine coupled to Vitamin D compared to children who received the isolated vaccine ( $\mathrm{p}=0.001$ with the cross tabulation method).

\section{Discussion}

Children are a sensitive target of infection, especially at a young age and after the weaning of maternal feeding which provides important protection; Children aged 2 - 5 years are most prone to infections due to their introduction to preschool/ nursery environments.

Table 1. The distribution of children.

\begin{tabular}{cccccc}
\hline & & \multicolumn{2}{c}{ FLU } & \multicolumn{2}{c}{ FLU \& VIT D } \\
\cline { 3 - 6 } & & Mean & Count & Mean & Count \\
\hline \multirow{2}{*}{ Age } & 4 & & 4 & \\
& Weight & 17 & & 18 & \\
Gender & MALE & & $80(53.3 \%)$ & & $101(51.3 \%)$ \\
& FEMALE & & $70(46.7 \%)$ & & $96(48.7 \%)$ \\
\hline
\end{tabular}


Numerous pediatricians recommend flu vaccine in order to decrease the rate of infection and its sequelae, the vaccine having shown positive results over the years.

Breast milk and formula lack Vitamin D. Therefore, supplementation is essential and needed. It is currently a subject of interest considering its increasing role recently in the immune system, vascular among many others. The European recommendations propose a supplementation of 100,000 UI during childhood and 200,000 UI during teenage years especially in winter season.

The aim of our study was to look for a synergistic effect of influenza vaccine combined with vitamin D. Our sample size consisted of 347 previously healthy children between 2 - 5 years, selected from three different out patients clinics across Lebanon taking into consideration variations in climate, altitude, eating habits and other factors.

The "Third National Health and Nutrition Examination Survey" revealed a significant association between vitamin D and upper respiratory tract infections [1]. However, in our study, patients who had upper respiratory tract infections had a lower average weight when compared to patients who did not have any upper respiratory tract infection.

A single Japanese study showed a decrease in the risk of children having influenza after supplementation in vitamin D. In fact, a daily dose of 1200 UI of vitamin D was administered over the course of 3 months to $50 \%$ of children aged 6 to 15 , compared to a control group. The study was a double blinded, randomized, performed in the faculty of medicine at the Jikel University in Tokyo [3].

In India, a study done on 150 children aged 2 to 5 years (similar to our group) divided into 2 groups, revealed a relationship between vitamin $\mathrm{D}$ deficiency and severe lower respiratory tract infections. A direct relationship between low dose of vitamin $\mathrm{D}$ and pneumonia was also mentioned [4].

Combining vitamin $\mathrm{D}$ and influenza vaccine can play an active role in the innate and acquired immune system by up-regulation of anti-microbial peptides, including defensins, and consequently protecting children against lower respiratory tract infections. Defensins would inhibit infections by blocking the hemagglutinin and the membrane fusion [5] [6].

Children having asthma are predisposed to bronchitis and pneumonia compared to the general population. The influenza vaccine protects against the influenza virus and vitamin $\mathrm{D}$ lowers the inflammatory response by regulating the production of cytokines responsible of bronchial hyper-reactivity therefore the administration of Vitamin D would reduce according to several studies asthma attacks as well as the frequency of respiratory infections especially during winter season [3] [7]. In fact, a low dose of vitamin D is associated with the presence of allergens, therefore it is proportional to asthma severity [8] The presence of vitamin $\mathrm{D}$ receptors (VDK) at the surface of macrophages, $\mathrm{B}, \mathrm{T}$ and Natural Killer lymphocytes [9], justify its role in the immune system by converting immune system into active metabolite. 
Not dividing children into subgroups considering factors such as prematurity, socio-economic status, living area, siblings, iron deficiency anemia, gastroesophageal reflux disease that may play a role in recurrent respiratory infection might be a limitation in our study. However, our patients were healthy children and criteria of exclusion mentioned above were used.

The Other limitation can be the sample size. In addition, and serum concentrations of 25-hydroxyvitamin D would have been useful to better correlate with the results of the study.

\section{Conclusions and Perspectives}

The WHO on an international level and the State on a national level recommend flu vaccination to any person at risk, specifically young children with risk factors of infections.

The American Academy of Pediatrics and the various Pediatric societies (European, Lebanese, etc.) recommend vitamin $\mathrm{D}$ supplementation, in order to cover vitamin $\mathrm{D}$ deficiency and due to its multiple benefits.

Our study proposes to combine two beneficial molecules proved to have a synergistic effect and represents a promising strategy in public health that we are publishing in hopes of encouraging more studies and eventually promoting the use of this coupling around the world.

\section{References}

[1] Cannell, J.J., Zasloff, M., Garland, C.F., Scragg, R. and Giovannucci, E. (2008) On the Epidemiology of Influenza. Virology Journal, 5, 29.

https://doi.org/10.1186/1743-422X-5-29

[2] Urashima, M., Segawa, T., Okazaki, M., Kurihara, M., Wada, Y. and Ida, H. (2010) Randomized Trial of Vitamin D Supplementation to Prevent Seasonal Influenza A in Schoolchildren. American Journal of Clinical Nutrition, 91, 1255-1260. https://doi.org/10.3945/ajcn.2009.29094

[3] Jeffery, L.E., Burke, F., Mura, M., Zheng, Y., Qureshi, O.S., Hewison, M., Walker, L.S., Lammas, D.A., Raza, K. and Sansom, D.M. (2009) 1,25-Dihydroxyvitamin D3 and IL-2 Combine to Inhibit T Cell Production of Inflammatory Cytokines and Promote Development of Regulatory T Cells Expressing CTLA-4 and FoxP3. Journal of Immunology, 183, 5458-5467. https://doi.org/10.4049/jimmunol.0803217

[4] Wayse, V., Yousafzai, A., Mogale, K. and Filteau, S. (2004) Association of Subclinical Vitamin D Deficiency with Severe Acute Lower Respiratory Infection in Indian Children under 5 y. European Journal of Clinical Nutrition, 58, 563-567. https://doi.org/10.1038/sj.ejcn.1601845

[5] Leikina, E., Delanoe-Ayari, H., Melikov, K., Cho, M.S., Chen, A., Waring, A.J., Wang, W., Xie, Y., Loo, J.A., Lehrer, R.I. and Chernomordik, L.V. (2005) Carbohydrate-Binding Molecules Inhibit Viral Fusion and Entry by Crosslinking Membrane Glycoproteins. Nature Immunology, 6, 995-1001. https://doi.org/10.1038/ni1248

[6] Doss, M., White, M.R., Tecle, T., Gantz, D., Crouch, E.C., Jung, G., Ruchala, P., Waring, A.J., Lehrer, R.I. and Hartshorn, K.L. (2009) Interactions of Alpha-, Beta-, and Theta-Defensins with Influenza A Virus and Surfactant Protein D. Journal of Immunology, 182, 7878-7887. https://doi.org/10.4049/jimmunol.0804049 
[7] Bahar-Shany, K., Ravid, A. and Koren, R. (2010) Upregulation of MMP-9 Production by TNFalpha in Keratinocytes and Its Attenuation by Vitamin D. Journal of Cellular Physiology, 222, 729-737.

[8] Brehm, J.M., Celedon, J.C., Soto-Quiros, M.E., Avila, L., Hunninghake, G.M., Forno, E., Laskey, D., Sylvia, J.S., Hollis, B.W., Weiss, S.T. and Litonjua, A.A. (2009) Serum Vitamin D Levels and Markers of Severity of Childhood Asthma in Costa Rica. American Journal of Respiratory and Critical Care Medicine, 179, 765-771. https://doi.org/10.1164/rccm.200808-13610C

[9] Hannoun, C. (2004) La grippe: Conceptions actuelles. Libbey Eurotext, Paris. 